Epidemiology and Psychiatric Sciences

cambridge.org/eps

\section{Editorials in This Issue}

Cite this article: Fiorillo A (2019). The complexity of vulnerability to psychosis. Epidemiology and Psychiatric Sciences 28, 138-139. https://doi.org/10.1017/ S2045796018000690

Received: 25 October 2018 Accepted: 26 October 2018

First published online: 29 November 2018

Key words:

Psychosis; risk factors; schizophrenia; adolescence

Author for correspondence: Andrea Fiorillo, E-mail: andrea.fiorillo@ unicampania.it

\title{
The complexity of vulnerability to psychosis
}

\section{Andrea Fiorillo}

Department of Psychiatry, University of Campania 'L. Vanvitelli', Naples, Italy

Schizophrenia is one the most puzzling mental diseases (Sullivan, 2012). Our understanding of its aetiopathological mechanisms is still far from being conclusive (Kendler, 2012). It is now being conceptualised as a neurodevelopmental rather than as a neurodegenerative illness, as it was conceived for decades (Weinberger, 2017), and psychotic disorders would lie on a continuum, from mild psychotic experiences observed in the general population to frank psychotic episodes. Recent studies have pointed out the role of a dopamine dysregulation in the mesostriatal circuit (Howes and Kapur, 2009) with an 'aberrant salience hypothesis' (Howes and Nour, 2016). According to this hypothesis, the dopamine release would result in an overattribution of meaning and motivational value (incentive salience) to irrelevant environmental events. Psychotic symptoms would be the final pathway of a complex dysregulation (Craddock and Owen, 2010), and their appearance would depend on a multitude of genetic and environmental factors that interact at various levels and in various modalities (Radua et al., 2018). As regards genetic liability, recent genome-wide association studies have identified a total of 108 conservatively defined loci that contain common risk alleles, and which meet genome-wide significance (Schizophrenia Working Group of the Psychiatric Genomics Consortium, 2014).

As regards environmental factors, higher rates of schizophrenia have been found in ethnic minority groups (Jongsma et al., 2018), in persons who are heavy cannabis smokers (Marconi et al., 2016), in those who suffered from severe childhood traumas, in persons who have been reared in highly deprived settings (O'Donoghue et al., 2016). Recent years have witnessed a renewed interest in social determinants of mental disorders, probably because neurobiological studies failed to identify the genetic variables or neurobiological pathways of mental disorders (De Rosa et al., 2018).

The finding that many individuals who are at high risk of developing psychosis actually do not develop a full-blown syndrome is consistent with the hypothesis that schizophrenia results from the interaction of environmental, sociocultural, genetic, neuropsychological and neurodevelopmental factors (Fusar-Poli et al., 2017). The identification of risk factors associated with psychosis is therefore essential for improving our understanding and early detection of vulnerable individuals, and to propose tailored interventions for sufferers. In fact, the delivery of intervention as early as possible has become one of the priorities for mental health professionals and other stakeholders involved in mental health care (Fiorillo et al., 2013; Malla et al., 2017).

The role of vulnerability factors to psychosis has been addressed in two editorials in this issue of Epidemiology and Psychiatric Sciences.

Psychosocial disability represents a frequent prodromal sign of psychosis, which exposes the patients to long-term social marginalisation, economic disadvantages and reduces the effectiveness of available treatments. Several longitudinal catamnestic studies have found that psychosocial disability is already present in the first 3-5 years of the disorder, which is considered to be a 'critical period' amenable to change if effective treatments are provided (Birchwood and Fiorillo, 2000). In the first Editorial Griffiths et al. (2018) highlighted the need to develop multi-level psychosocial interventions in order to reduce psychosocial disability not only in ultra-high-risk patients or in patients with a full-blown diagnosis of schizophrenia, but also in the general population and in NEET people.

In the other Editorial, the role of migration as a relevant risk factor for psychosis is discussed (Dykxhoorn and Kirkbride, 2018). In particular, a wide heterogeneity between different migrant groups has been found in epidemiological studies, thus providing a model for differentiating the complex aetiological pathways of schizophrenia. These epidemiological findings have been recently confirmed by neuroscience studies, which found that migration and minority status are associated with structural and functional brain differences relevant to psychosis. The authors suggest the need to implement multidisciplinary population-based studies in order to define the role of other variables in shaping the risk for psychosis, beyond the migration process itself. In fact, migration is becoming very frequent and, therefore, is essential to understand the specific role of migration as risk factor for schizophrenia, in order to: a) develop a predictive model of transition into psychosis, b) propose adequate preventive strategies and c) reshape early intervention services (Fiorillo and Maj, 2018).

The complexity of schizophrenia is well represented in these two editorials, which clearly point out the need for an interdisciplinary approach to this disorder. What is needed in psychiatric practice is a global and integrated approach, which include screening procedures for 
individuals reporting specific vulnerabilities, in order to tailor and individualise treatment approaches on patients' needs (Slade, 2017; Puschner, 2018).

Acknowledgement. None.

Financial support. This research received no specific grant from any funding agency, commercial or not-for-profit sectors.

Conflict of interest. None.

\section{References}

Birchwood M and Fiorillo A (2000) The critical period for early intervention. Psychiatric Rehabilitation Skills 4, 182-198.

Craddock N and Owen MJ (2010) The Kraepelinian dichotomy - going, going... but still not gone. The British Journal of Psychiatry 196, 92-95.

De Rosa C, Sampogna G, Luciano M, Del Vecchio V, Fabrazzo M and Fiorillo A (2018) Social versus biological psychiatry: it's time for integration! International Journal of Social Psychiatry 1, 20764017752969. [Epub ahead of print].

Dykxhoorn J and Kirkbride J (2018) Psychoses sans Frontieres: towards an interdisciplinary understanding of psychosis risk amongst migrants and their descendants. Epidemiology and Psychiatric Sciences In press.

Fiorillo A and Maj M (2018) The role of psychiatry in modern medicine. International Review of Psychiatry 30, 169-175.

Fiorillo A, Luciano M, Del Vecchio V, Sampogna G, Obradors-Tarragó C and Maj M (2013) Priorities for mental health research in Europe: a survey among national stakeholders' associations within the ROAMER project. World Psychiatry 12, 165-170.

Fusar-Poli P, Tantardini M, De Simone S, Ramella-Cravaro V, Oliver D, Kingdon J, Kotlicka-Antczak M, Valmaggia L, Lee J, Millan MJ, Galderisi S, Balottin U, Ricca V and McGuire P (2017) Deconstructing vulnerability for psychosis: meta-analysis of environmental risk factors for psychosis in subjects at ultra high-risk. European Psychiatry 40, 65-75.

Griffiths SL, Wood SJ and Birchwood M (2018) Vulnerability to psychosocial disability in psychosis. Epidemiology and Psychiatric Sciences In press.
Howes OD and Kapur S (2009) The dopamine hypothesis of schizophrenia: version III - the final common pathway. Schizophrenia Bulletin 35, 549-562.

Howes OD and Nour MM (2016) Dopamine and the aberrant salience hypothesis of schizophrenia. World Psychiatry 15, 3-4.

Jongsma HE, Gayer-Anderson C, Lasalvia A, Quattrone D, Mulè A, Szöke A, Selten JP, Turner C, Arango C, Tarricone I, Berardi D, Tortelli A, Llorca PM, de Haan L, Bobes J, Bernardo M, Sanjuán J, Santos JL, Arrojo M, Del-Ben CM, Menezes PR, Velthorst E, Murray RM, Rutten BP, Jones PB, van Os J, Morgan C, Kirkbride JB; European Network of National Schizophrenia Networks Studying Gene-Environment Interactions Work Package 2 (EU-GEI WP2) Group (2018) Treated incidence of psychotic disorders in the multinational EU-GEI study. JAMA Psychiatry 75, 36-46.

Kendler KS (2012) The dappled nature of causes of psychiatric illness: replacing the organic-functional/hardware-software dichotomy with empirically based pluralism. Molecular Psychiatry 17, 377-388.

Malla A, Shah J and Lal S (2017) Advances and challenges in early intervention in psychosis. World Psychiatry 16, 274-275.

Marconi A, Di Forti M, Lewis CM, Murray RM and Vassos E (2016) Meta-analysis of the association between the level of cannabis use and risk of psychosis. Schizophrenia Bulletin 42, 1262-1269.

O’Donoghue B, Roche E and Lane A (2016) Neighbourhood level social deprivation and the risk of psychotic disorders: a systematic review. Social Psychiatry and Psychiatric Epidemiology 51, 941-950.

Puschner B (2018) Peer support and global mental health. Epidemiology and Psychiatric Sciences 27, 413-414.

Radua J, Ramella-Cravaro V, Ioannidis JP, Reichenberg A, Phiphopthatsanee N, Amir T, Yenn Thoo H, Oliver D, Davies C, Morgan C, McGuire P, Murray RM and Fusar-Poli P (2018) What causes psychosis? An umbrella review of risk and protective factors. World Psychiatry 17, 49-66.

Schizophrenia Working Group of the Psychiatric Genomics Consortium (2014) Biological insights from 108 schizophrenia-associated genetic loci. Nature 511, 421-427.

Slade M (2017) Implementing shared decision making in routine mental health care. World Psychiatry 16, 146-153.

Sullivan PF (2012) Schizophrenia as a pathway disease. Nature Medicine 18, $210-211$.

Weinberger D (2017) The neurodevelopmental origins of schizophrenia in the penumbra of genomic medicine. World Psychiatry 16, 225-226. 